\title{
Retraction: The helminth product ES-62 protects against septic shock via Toll-like receptor 4-dependent autophagosomal degradation of the adaptor MyD88
}

Padmam Puneet, Mairi A McGrath, Hwee Kee Tay, Lamyaa Al-Riyami, Justyna Rzepecka, Shabbir M Moochhala, Shazib Pervaiz, Margaret M Harnett, William Harnett \& Alirio J Melendez

Nat. Immunol. 12, 344-351 (2011); published online 27 February 2011; retracted 24 June 2011

The authors wish to note the following. Irregularities have been identified in some of the figures in this paper. The conclusions drawn from these data, that ES-62 protects against the development of pathology in the sepsis models and results in the induction of autophagy in macrophages, cannot be made. As these conclusions constitute major components of the paper, we wish to retract this paper.

\section{Addendum: Defeating sepsis by misleading MyD88}

Katherine A Smith \& Rick M Maizels

Nat. Immunol. 12, 284-286 (2011); published online 21 April 2011; addendum published after print 24 June 2011

The article to which this News and Views refers has been retracted (http://www.nature.com/ni/journal/v12/n4/abs/ni.2004.html). Nature Immunology wishes to inform readers that some of the comments made in this News and Views, although made in good faith based on the article's conclusions, may no longer be valid. 